\title{
Metastatic Human Epidermal Growth Factor Receptor 2-Positive Breast Cancer: Current Treatment Standards and Future Perspectives
}

\author{
Clemens Dormann \\ Interne I: Medizinische Onkologie und Hämatologie, Ordensklinikum Linz Barmherzige Schwestern, Linz, Austria
}

\author{
Keywords \\ Tucatinib · Neratinib $\cdot$ Pyrotinib $\cdot$ Trastuzumab deruxtecan . \\ Margetuximab $\cdot$ Advanced breast cancer
}

\begin{abstract}
Background: The basis of improved systemic therapy for inoperable or metastatic human epidermal growth factor receptor 2 (HER2)-positive breast cancer is formed by HER2targeting monoclonal antibodies. Dual HER2 blockade with pertuzumab and trastuzumab in combination with docetaxel in previously untreated patients, and trastuzumab emtansine (T-DM1, an antibody-drug conjugate [ADC] consisting of trastuzumab, a linker and a cytotoxic payload) after prior trastuzumab therapy have demonstrated progression-free survival (PFS) and overall survival (OS) superior to what was achieved with the previous treatment routine. Therefore, pertuzumab and trastuzumab with chemotherapy (preferably with a taxane) and T-DM1 are considered the current standard of care in the first- and second-line settings, respectively. For later lines of therapy, no uniformly recognized standard of care has been defined. Accepted options include treatment with trastuzumab beyond progression, in combination with a broad variety of single-agent chemotherapies used sequentially, or lapatinib (an HER2-targeting tyrosine kinase inhibitor [TKI]) in combination with either trastuzumab or capecitabine. However, most of these options have not been formally tested in patients receiving the current standard of care therapy for metastatic disease. Summary: In patients previously treated with today's standard of care, including a significant subgroup with untreated or progress-
\end{abstract}

ing brain metastases, the combination of tucatinib, a novel HER2-targeting TKI, with trastuzumab and capecitabine, demonstrates a clinically meaningful improvement in PFS and OS when compared to placebo with trastuzumab and capecitabine. Neratinib, another HER2 TKI, in combination with capecitabine, compared to lapatinib and capecitabine, as well as margetuximab, an HER2-directed monoclonal antibody with a fragment c $(\mathrm{Fc})$ domain engineered to enhance immune activation, compared to trastuzumab, both combined with the investigator's choice of chemotherapy, showed a statistically significantly longer PFS. However, not all patients in the respective trials had received pertuzumab and T-DM1 prior to enrollment and, so far, no improvement in OS has been demonstrated. After a median of 6 prior lines of therapy, trastuzumab deruxtecan (T-DXd), a novel ADC, showed a meaningful overall response and PFS. Although the safety profile was generally manageable, treatment-related interstitial lung disease (ILD) might pose a challenge in routine practice. Pyrotinib, another HER2 TKI, was evaluated in combination with capecitabine in patients after prior exposure to trastuzumab when pertuzumab and T-DM1 were not available. In this setting, PFS was better than with lapatinib and capecitabine. Key Messages: In 2020, pertuzumab and trastuzumab with taxane-based chemotherapy in the first line, and T-DM1 in the second line, remain the standard of care. Tucatinib, neratinib, margetuximab, and T-DXd expand the armamentarium for treatment beyond the second line. Pyrotinib might be another option, especially for patients, who do not have access to pertuzumab and T-DM1.

(c) 2020 S. Karger AG, Basel 


\section{First-Line Treatment: Pertuzumab}

Unlike trastuzumab, which binds to subdomain IV of the human epidermal growth factor receptor 2 (HER2) extracellular domain, pertuzumab, another humanized monoclonal antibody, binds to subdomain II, which prevents HER2 from dimerizing with other ligand-activated HER receptors, most notably HER3. Like trastuzumab, pertuzumab stimulates antibody-dependent, cell-mediated cytotoxicity. Due to the different binding and complementary mechanisms of action, the combination of trastuzumab and pertuzumab provides a more comprehensive blockade of HER2 signaling and results in greater antitumor activity than either agent alone [1-5].

In CLEOPATRA, a double-blind, phase 3 trial, 808 patients with locally recurrent, unresectable, or metastatic HER2-positive breast cancer who had not received chemotherapy or biologic therapy for advanced disease, were randomly assigned 1:1 to pertuzumab at a loading dose of $840 \mathrm{mg}$, followed by $420 \mathrm{mg}$ intravenously every 3 weeks or placebo, both combined with standard-dose trastuzumab and docetaxel. One line of endocrine therapy for metastatic disease and chemotherapy, with or without trastuzumab, for early breast cancer, if completed 12 months prior to the diagnosis of advanced disease, were permitted. Patients with central nervous system (CNS) metastases were excluded from the study.

Of the randomized patients, 408 (50.5\%) had hormone receptor (HR)-negative cancers, 630 (78.0\%) had visceral involvement, and 376 (46.5\%) had received treatment in an early disease setting (including trastuzumab in 88 [10.9\%] cases).

In the pertuzumab-combination group, progressionfree survival (PFS) assessed by independent review, the trial's primary end point, was significantly improved when compared to the placebo combination after a median follow-up of 19.3 months (median PFS 18.5 vs. 12.4 months; hazard ratio $0.62 ; p<0.001$ ) [6]. At the second interim analysis (data maturity $69 \%$ ), after a median follow-up of 30 months, median overall survival [OS] was 37.6 months in the control arm but had not been reached in the pertuzumab group (hazard ratio $0.66 ; p=0.0008$ ). The hazard ratio crossed the prespecified stopping boundary for efficacy, thus demonstrating a significant improvement in survival with the addition of pertuzumab [7]. At the prespecified, descriptive end-of-study analysis median OS, based on the intention-to-treat (ITT) population, was 57.1 months in the pertuzumab group and 40.8 months in the placebo group (including $50(12.3 \%)$ patients who had crossed over to the pertuzumab group after the second interim analysis) after a median follow-up of 99 months. The 8-year landmark OS rates were $37 \%$ in the pertuzumab group and $23 \%$ in the control arm [8].
The trial's safety population comprised 804 treated patients. Pre-crossover, the median number of treatment cycles was 24.0 in the pertuzumab group and 15.0 in the placebo group. Post-crossover, the median number of pertuzumab cycles was 42.0. Docetaxel was administered for a median of 8 cycles in both arms. Thirty-nine $(9.6 \%)$ patients withdrew from all study medication pre-crossover due to an adverse event (AE) in the pertuzumab group, and $24(6.1 \%)$ discontinued in the placebo group. Febrile neutropenia was the most common treatment-related serious AE ( $11.3 \%$ in the pertuzumab group vs. $4.8 \%$ in the placebo group). Grade $\geq 3$ diarrhea and rash occurred more frequently in the pertuzumab-combination arm (9.6 vs. 5.1\% and 3.7 vs. $1.5 \%$, respectively). All-grade left ventricular dysfunction was comparable between arms ( $7.8 \%$ with pertuzumab vs. $8.6 \%$ with placebo). Five patients in the pertuzumab group and 6 in the placebo group died from treatment-related AEs [8].

Dual HER2-blockade with pertuzumab and trastuzumab was also evaluated in combination with vinorelbine, capecitabine, paclitaxel, nab-paclitaxel, and endocrine therapy [9-12].

Based on these results, the combination of pertuzumab and trastuzumab with chemotherapy, preferably a taxane, is considered the standard of care for the first-line treatment of unresectable or metastatic HER2-positive breast cancer $[13,14]$.

\section{Second-Line Treatment: Trastuzumab Emtansine}

Trastuzumab emtansine (T-DM1) is an antibody-drug conjugate (ADC) consisting of trastuzumab, which maintains its HER2-targeted antitumor properties, a nonreducible thioether linker (SMCC), and DM1 (a derivate of maytansine), a potent microtubule inhibitor, as the cytotoxic payload. The primary active metabolite, lysineMCC-DM1, does not readily cross the plasma membranes of neighboring cells, and thus does not elicit a cytotoxic bystander effect. T-DM1 has a drug-to-antibody ratio of 3 to 3.6 [15-17].

In EMILIA, an open-label, phase 3 trial, 991 patients with HER2-positive, unresectable, locally advanced or metastatic breast cancer, who had previously been treated with trastuzumab and a taxane and were progressing on or after the most recent therapy for advanced disease or within 6 months after treatment for early-stage disease, were randomized 1:1 to T-DM1 $3.6 \mathrm{mg} / \mathrm{kg}$ intravenously every 3 weeks, or lapatinib $1,250 \mathrm{mg}$ orally once daily continuously plus standard-dose capecitabine. Previous exposure to T-DM1, lapatinib, or capecitabine was prohibited. Patients with asymptomatic or treated brain metastases could be included. 
Of all randomized patients, 426 (43.0\%) had HR-negative cancers and $669(67.5 \%)$ had visceral disease; 118 (11.9\%) were treated in the first-line setting, 361 (36.4\%) in the second-line setting, and the remainder in a later line.

Treatment with T-DM1 significantly improved both coprimary end points when compared to the control arm: (1) PFS as assessed by independent review after a median follow-up of approximately 13 months (median PFS 9.6 vs. 6.4 months; hazard ratio $0.65 ; p<0.001)$, and (2) OS at the second interim analysis (data maturity 50\%) after a median follow-up of approximately 19 months (median OS 30.9 vs. 25.1 months; hazard ratio 0.68 ; $p<$ 0.001 ), which crossed the stopping boundary for efficacy [18]. After the second interim analysis, 136 (27.4\%) patients in the control arm crossed over to T-DM1. Of all patients originally allocated to T-DM1, 254 (51.3\%) received capecitabine and 241 (48.7\%) were treated with lapatinib, separately or in combination, after discontinuation of the study drug. At the final descriptive analysis, after a median follow-up of 41.9 months in the control group and 47.8 months in the T-DM1 group, median OS in the ITT population was longer with T-DM1 than with lapatinib and capecitabine (29.9 vs. 25.9 months; hazard ratio 0.75 ) [19].

The trial's safety population comprised 978 patients who received at least 1 dose of study treatment. Due to AEs, capecitabine was discontinued in 53 (10.9\%) patients, lapatinib in 42 (8.6\%) and T-DM1 in 49 (10.0\%). Grade $\geq 3$ AEs as well as serious AEs were more frequent in the control arm than in the T-DM1 arm (59.6 vs. $47.6 \%$ and 20.3 vs. $18.6 \%$, respectively). The most commonly reported $\mathrm{AE}$ grade $\geq 3$ with T-DM1 was thrombocytopenia $(14.3 \%)$. Three patients receiving T-DM1 and 2 receiving capecitabine + lapatinib died from treatment-related AEs [19].

As pertuzumab was not routinely available at the time of study enrollment, its prior use was not specified in the protocol and it was therefore not systematically documented. However, only a small number of patients in EMILIA are suspected to have received previous pertuzumab [19]. Since the approval of pertuzumab and T-DM1, several studies have investigated T-DM1's efficacy after prior treatment with pertuzumab, trastuzumab, and taxane-based chemotherapy. The median PFS of second-line T-DM1 was 6.3 months for 77 patients in the Gruppo Italiano Mammella (GIM) 14/BIOMETA study (a retrospective/prospective multicenter study on treatment patterns and outcomes of patients with metastatic breast cancer) and 10.5 months for 135 patients in a real-life setting $[20,21]$.

Based on these results, T-DM1 is considered the standard of care for the second-line treatment of unresectable or metastatic HER2-positve breast cancer [13, 14].

\section{Novel Treatment Options beyond the Second Line}

\section{Tucatinib}

Tucatinib is an orally available, reversible tyrosine kinase inhibitor (TKI), highly selective for the kinase domain of HER2, with only marginal activity against epidermal growth factor receptor (EGFR) [22, 23].

HER2CLIMB, a double-blind, pivotal phase 3 trial included 612 patients with advanced HER2-positive breast cancer, who had been treated with trastuzumab, pertuzumab, and T-DM1 prior to study entry. Previous therapy with capecitabine or an HER2-targeted TKI for metastatic disease was prohibited. However, patients who had received lapatinib $>12$ months before initiating a trial regimen, were eligible. Enrollment of patients with brain metastases was allowed unless they were in need of immediate local intervention, in which case they could receive local therapy and be enrolled subsequently. Patients were excluded when signs of leptomeningeal spread were found on the mandatory contrast magnetic resonance imaging (MRI) at baseline.

All patients were randomly assigned 2:1 to either tucatinib, $300 \mathrm{mg}$ orally twice daily continuously, or matching placebo, in combination with standard-dose trastuzumab and capecitabine. Of these, 236 (38.6\%) patients had HRnegative tumors and 220 (35.9\%) had de novo metastatic disease. Prior to inclusion, patients had received a median of 4 lines of systemic therapy, 3 of which were for metastatic disease.

The trial's primary end-point population comprised the first 480 patients randomized. Within this group, the addition of tucatinib significantly reduced the risk of death or disease progression (hazard ratio $0.54 ; p<0.001$ ). Median PFS, as evaluated by blinded independent central review (BICR), was 7.8 months in the tucatinib-combination group and 5.6 months in the placebo-combination group. The median duration of follow-up in the total population was 14.0 months.

At the first interim analysis, median OS, assessed in the entire study population, was 21.9 months in the tucatinib-combination group versus 17.4 months in the control group (hazard ratio $0.66 ; p=0.005$ ), crossing the predefined, multiplicity-adjusted margin of significance [24].

A subset of special interest were patients with brain metastases, as early data implicated promising activity in this group [25]. HER2CLIMB enrolled 291 patients with CNS involvement, i.e., $47.5 \%$ of the total population. Of these, $66(22.7 \%)$ had untreated, $108(37.1 \%)$ had treated but progressing, and $117(40.2 \%)$ had treated and stable brain metastases. Two hundred and four (70.1\%) patients had received prior radiotherapy and only $46(15.8 \%)$ had had prior surgery [26]. The addition of tucatinib led to an improvement in median PFS in patients with brain me- 
tastases from 5.4 months in the placebo-combination group to 7.6 months (hazard ratio $0.48 ; p<0.001$ ) [24]. In exploratory analyses on all patients with brain metastases, median CNS-PFS, i.e., the time from randomization to investigator-assessed intracranial progression or death, was 9.9 months in the tucatinib-combination group and 4.2 months in the placebo-combination group (hazard ratio 0.32 ; 95\% confidence interval [CI] 0.22-0.48) and median OS was 18.1 months and 12.0 months, respectively (hazard ratio 0.58 ; 95\% CI 0.40-0.85) [26].

The trial's safety population consisted of 601 patients who received at least 1 dose of any study drug or placebo. Among these, median duration of exposure to tucatinib or placebo was 5.8 and 4.4 months, respectively. A slightly higher rate of grade $\geq 3$ AEs was observed with the addition of tucatinib (55.2 vs. $48.7 \%$ ). AEs led to the discontinuation of tucatinib in $23(5.7 \%)$ patients and of placebo in $6(3.0 \%)$ patients. Diarrhea was the most common $\mathrm{AE}$ in both groups $(80.9 \%$ with tucatinib vs. $53.3 \%$ with placebo); however, grade $\geq 3$ events were rare and comparable between groups (12.9 and 8.6\%). AEs caused the death of 6 patients in the tucatinib-combination group and 5 in the placebo-combination group [24].

\section{Neratinib}

Neratinib is an oral, irreversible, pan-HER TKI, with activity against EGFR (HER1), HER2, and HER4 [27].

In NALA, an open-label, phase 3 trial, 621 patients with HER2-positive metastatic breast cancer, who had received $\geq 2$ prior HER2-directed regimens for advancedstage disease, were randomized 1:1 to neratinib $240 \mathrm{mg}$ once daily continuously with capecitabine $1,500 \mathrm{mg} / \mathrm{m}^{2}$, or lapatinib $1,250 \mathrm{mg}$ once daily continuously with capecitabine $2,000 \mathrm{mg} / \mathrm{m}^{2}$. In both arms, capecitabine was administered on days 1-14 of 21-day cycles. In the neratinib-combination group, antidiarrheal prophylaxis with loperamide was mandatory for the first cycle and at the investigator's discretion thereafter. Prior exposure to capecitabine or any HER2-targeted TKI or concurrent endocrine therapy was not permitted. Patients with asymptomatic or stable (treated or untreated) brain metastases could be enrolled.

Of all randomized patients, 254 (40.9\%) had HR-negative tumors, 529 (85.2\%) had visceral metastases, and $430(69.2 \%)$ had received 2 prior lines of therapy as the rest were treated in a more advanced setting. Two hundred and fifteen (34.6\%) patients had been previously exposed to trastuzumab, pertuzumab and T-DM1, but a large proportion, i.e., 237 (38.2\%) patients, had only been treated with trastuzumab combined with chemotherapy or endocrine therapy before enrollment.

After a median follow-up of 29.9 months, the risk of centrally assessed disease progression or death from any cause, one of the trial's coprimary end points, was sig- nificantly reduced with the neratinib combination compared to with the lapatinib combination (hazard ratio $0.76 ; p=0.0059)$. As the proportional-hazards assumption was violated, a restricted-means analysis was performed with restriction at 24 months. This supported the primary analysis, demonstrating a mean PFS difference of 2.2 months in favor of the neratinib combination $(8.8$ months vs. 6.6 months; $p=0.0003)$. Significant interactions were observed in 2 subgroups, indicating greater benefit for patients with nonvisceral or HR-negative disease. OS, the second coprimary end point, was not significantly improved (hazard ratio $0.88 ; p=0.2086$ ). However, as PFS was significant at the split a level (an overall type 1 error rate of 0.01 for PFS), the study was considered positive. The overall cumulative incidence of intervention for CNS disease was $22.8 \%$ for neratinib and $29.2 \%$ for lapatinib (Gray's test for equality, $p=0.043$ ).

The trial's safety population comprised 614 patients who received $\geq 1$ dose of investigational treatment. The median duration of exposure to neratinib or lapatinib was 5.7 and 4.4 months, respectively. Treatment-emergent AEs led to discontinuation of any study drug in 42 (13.9\%) patients in the neratinib-combination arm and $56(18.0 \%)$ in the lapatinib-combination arm. Due to AEs, TKI dose reduction occurred in $9.9 \%$ in the neratinib group and $10.6 \%$ in the lapatinib group, and TKI hold in 50.2 and $46.6 \%$, respectively. Capecitabine was reduced more often in the lapatinib arm, which could be attributed to the higher dose used (28.6 vs. 20.8\%). Grade $\geq 3$ and serious treatment-emergent AEs were reported in 60.7 and $34.0 \%$ in the neratinib-combination group and in 60.5 and $29.9 \%$ in the lapatinib-combination group. Diarrhea was the most common $\mathrm{AE}$ in both groups. Despite primary prophylaxis with loperamide, all-grade and grade 3 diarrhea occurred more frequently in the neratinib group, 83.2 versus $66.2 \%$ and 24.4 vs. $12.5 \%$, respectively. However, the duration of grade $3 \mathrm{di}$ arrhea and treatment discontinuation due to diarrhea (2.6\% with neratinib vs. $2.3 \%$ with lapatinib) did not differ significantly between arms. One death was considered to be treatment-related and it occurred in the lapatinib-combination group [28].

\section{Margetuximab}

Margetuximab is a monoclonal antibody derived from 4D5, the parent antibody of trastuzumab. Margetuximab and trastuzumab bind the same epitope of HER2 with similar affinities and exhibit similar tumor-directed, effector cell-independent, antiproliferative activity in breast cancer cells in vitro in the absence of immune effectors. However, margetuximab's immunoglobulin (Ig)G1 Fc domain was engineered, compared to trastuzumab's wild-type domain, to increase binding to both, the higher-affinity $158 \mathrm{~V}$ and the lower-affinity $158 \mathrm{~F}$, alleles of 
cluster of differentiation (CD) 16A (Fc $\gamma$ RIIIA), a stimulatory receptor, and decrease binding to $\mathrm{CD} 32 \mathrm{~B}(\mathrm{Fc} \gamma \mathrm{RIIB})$, an inhibitory receptor. Both receptors are important for regulating antibody-dependent cell-mediated cytotoxicity (ADCC), a function of the innate immune system. Margetuximab demonstrated cytotoxicity in ADCC assays using effector cells from donors heterozygous or homozygous for the lower-affinity $158 \mathrm{~F}$ variant of CD16A superior to that of a trastuzumab surrogate with a wildtype $\mathrm{Fc}$ domain [29]. In a phase 1 trial involving patients with pretreated, HER2-positive cancers, margetuximab also potentiated adaptive immunity, including enhanced clonality of the T-cell repertoire and induction of HER2specific T-cell and B-cell responses [30].

In SOPHIA, an open-label, phase 3 trial, 536 patients with unresectable or metastatic HER2-positive breast cancer, who had received $\geq 2$ prior HER2-directed therapies including pertuzumab, and 1-3 lines in the metastatic setting, were randomized 1:1 to the investigator's choice of chemotherapy (capecitabine, eribulin, gemcitabine, or vinorelbine) combined with either margetuximab at $15 \mathrm{mg} / \mathrm{kg}$ intravenously every 3 weeks or standard-dose trastuzumab. Patients with treated and stable brain metastases could be enrolled.

Of all randomized patients, 200 (37.3\%) had HR-negative cancers; 489 (91.2\%) had previously been treated with T-DM1 and $92(17.2 \%)$ with lapatinib or another HER2-directed TKI. Three hundred and fifty-five (66.2\%) patients had received $\leq 2$ prior lines for metastatic disease as the rest were treated in a more advanced setting. All patients had been treated with trastuzumab and pertuzumab prior to enrollment: 501 (93.5\%) had thus been exposed to a taxane, 228 (42.5\%) to an anthracycline, and $259(48.3 \%)$ to endocrine therapy. Slightly fewer patients in the margetuximab group had received treatment for metastatic disease only (40.6 vs. $46.3 \%$ ).

PFS by central blinded analysis, one of the trial's coprimary end points, was prolonged in patients receiving margetuximab compared to those receiving trastuzumab (median PFS 5.8 vs. 4.9 months; hazard ratio $0.76 ; p=$ 0.033). In a planned exploratory analysis of $506(94.4 \%)$ patients with available genotyping information, PFS benefit appeared to be more pronounced in the 437 (86.4\%) patients harboring at least $1 \mathrm{CD} 16 \mathrm{~A} 158 \mathrm{~F}$ allele (median PFS 6.9 vs. 5.1 months; unstratified hazard ratio $0.68 ; p=$ 0.005), whereas the effects seemed to diminish in the 69 (13.6\%) patients with homozygous CD16A 158V alleles (median PFS 4.8 vs. 5.6 months; unstratified hazard ratio $1.78 ; p=0.11$ ). The relative improvement with margetuximab was consistent in CD16A $158 \mathrm{FF}$ and $158 \mathrm{FV}$ carriers [31]. No significant difference in OS, the sequentially assessed, coprimary end point, could be demonstrated at the planned first [31] and second [32] interim analyses (data maturity 41 and $70 \%$ ) or in a prespecified explor- atory analysis in the patients harboring at least $1 \mathrm{CD} 16 \mathrm{~A}$ $158 \mathrm{~F}$ allele $[31,32]$.

The trial's safety population comprised 529 patients who received study therapy [31]. Grade $\geq 3$, grade $\geq 3$ antibody-related, and serious AEs occurred in 53.8, 12.9, and $16.3 \%$ in the margetuximab group and in $52.6,8.3$, and $18.4 \%$ in the trastuzumab group. AEs leading to treatment discontinuation occurred in $8(3.0 \%)$ patients on margetuximab and in 7 (2.6\%) on trastuzumab [32]. The most common grade $\geq 3$ AEs were hematologic, i.e., neutropenia (19.3\% in the margetuximab arm vs. $11.3 \%$ in the control arm) [31]. Infusion-related reactions (IRR), an AE of special interest, occurred in $13.3 \%$ of patients on margetuximab, with $1.5 \%$ experiencing grade $\geq 3$ events, but in only $3.4 \%$ of patients in the control group (without any grade $\geq 3$ events), as all patients were pretreated with trastuzumab. However, as most of the IRRs were lowgrade and easily managed with premedication, only 2 $(0.8 \%)$ patients discontinued treatment in the margetuximab arm versus none in the trastuzumab arm. AEs caused the death of 3 patients in the margetuximab arm and 2 in the trastuzumab arm. However, none of these events was considered antibody-related [32].

\section{Trastuzumab Deruxtecan}

Trastuzumab deruxtecan (T-DXd) is an ADC consisting of a humanized, anti-HER2 monoclonal antibody with the same structure as trastuzumab, a tetrapeptidebased linker which is stable in plasma and selectively cleaved by cathepsins that are upregulated in tumor cells, and exatecan which is a potent new topoisomerase I inhibitor, as the cytotoxic payload. The released payload easily crosses the cell membrane, which potentially allows target-independent cytotoxic effects on neighboring tumor cells. However, systemic exposure is minimized because of exatecan's short half-life. T-DXd has a drug-toantibody ratio of 8, twice that of T-DM1 [33-38].

In DESTINY-Breast01, a two-part, open-label, phase 2 registration study, 253 patients with HER2-positive unresectable or metastatic breast cancer, who were pretreated with T-DM1, were enrolled. Patients with interstitial lung disease (ILD) or noninfectious pneumonitis were excluded. The first part of the study consisted of a pharmacokinetic and a dose-finding stage, including 65 and 54 patients, respectively. In part 2, the recommended dose of $5.4 \mathrm{mg} / \mathrm{kg}$ administered intravenously every 3 weeks was evaluated in 130 patients who had tumor progression on or after T-DM1, and in 4 patients who were intolerant to T-DM1 [39].

The primary end point population comprised $184 \mathrm{pa}-$ tients who received the recommended dose of T-DXd in both parts of the trial, including 24 (13.0\%) patients with treated and asymptomatic brain metastases. Among all patients, 83 (45.1\%) had HR-negative tumors, $169(91.8 \%)$ 
had visceral disease, 102 (55.4\%) had an Eastern Cooperative Oncology Group (ECOG) performance status of 0 , and 93 (50.5\%) had previously received an HER2-targeting TKI versus 14 (58.3\%), 24 (100\%), 15 (62.5\%), and $15(62.5 \%)$ of those with CNS involvement. All patients had been exposed to trastuzumab and T-DM1, with 66 (35.9\%) being primarily resistant to the ADC, and 121 (65.8\%) having been treated with pertuzumab, as patients had received a median of 6 lines of systemic therapy prior to enrollment $[39,40]$.

The trial's primary end point, the confirmed overall response rate (ORR) on independent central review, reached $60.9 \%$ after a median follow-up of 11.1 months. The disease control rate (DCR) was $97.3 \%$, the clinical benefit rate was $76.1 \%$, and the median duration of PFS was 16.4 months. Estimated OS was $86.2 \%$ at 12 months, while the median was not reached at data cut-off [39]. Of 24 patients with CNS involvement, confirmed ORR was $58.3 \%$, DCR was $91.7 \%$, and the median PFS was 18.1 months. Forty-eight $(26.1 \%)$ patients experienced disease progression including 4 patients with CNS progression, 2 of whom developed de novo brain metastases [40].

After a median treatment duration of 10.0 months in the primary end point population, $57.1 \%$ of the patients had at least 1 treatment-emergent $\mathrm{AE}$ grade $\geq 3,48.4 \%$ had a drug-related $\mathrm{AE}$ grade $\geq 3,22.8 \%$ had a serious $\mathrm{AE}$, and $12.5 \%$ had a drug-related serious AE. Treatmentemergent AEs led to discontinuation in 28 (15.2\%) patients, including 16 cases of ILD or pneumonitis. AEs caused dose reduction in $23.4 \%$ and interruption in $35.3 \%$. The most common grade $\geq 3$ AEs were hematologic, i.e., a decreased neutrophil count $(20.7 \%)$; febrile neutropenia occurred in only $1.6 \%(n=3)$ of cases; and gastrointestinal events (nausea in 7.6\%). As determined by an independent adjudication committee, 25 (13.6\%) patients had ILD related to the receipt of T-DXd, including 4 events that were fatal. At the time of data cut-off, 10 patients still had ongoing ILD [39].

\section{A Novel Option for Pretreated Patients without Access to Pertuzumab and T-DM1: Pyrotinib}

Pyrotinib is an oral, irreversible, pan-HER TKI with activity against EGFR (HER1), HER2, and HER4 [41].

In PHOEBE, an open-label, multicenter, phase $3 \mathrm{Chi}-$ nese study, 267 patients with HER2-positive metastatic breast cancer, pretreated with trastuzumab and taxanes and/or anthracyclines, who had received $\leq 2$ lines of chemotherapy for metastatic disease, were randomly assigned 1:1 to pyrotinib $400 \mathrm{mg}$ once daily continuously, or lapatinib $1,250 \mathrm{mg}$ once daily continuously, both in combination with standard-dose capecitabine. Prior ex- posure to capecitabine in the metastatic setting or an HER2-targeted TKI was not permitted. Patients with brain metastases were excluded.

Of all randomized patients, 146 (54.9\%) had HR-negative disease, 211 (79.3\%) had visceral metastases, and 69 (25.9\%) showed resistance to prior trastuzumab, which was defined as a relapse within 6 months after adjuvant trastuzumab and/or disease progression within 3 months of treatment for metastatic disease. Forty (15.0\%) patients had received trastuzumab for early- and advanced-stage disease. In the pyrotinib-combination group, slightly fewer had received it for metastatic disease (59.0 vs. $67.4 \%$ ), as more patients in this group were treated in a first-line setting ( 42.5 vs. $34.8 \%)$. Only $42(15.8 \%)$ patients had received 2 preceding lines of therapy for advanced-stage disease prior to enrollment.

After a median follow-up of 9.9 months, PFS assessed by BICR in the full analysis set of 266 treated patients (the trial's primary end point) was improved in the pyrotinibcombination group compared to the lapatinib-combination group (median PFS 12.5 vs. 6.8 months; hazard ratio $0.39 ; p<0.0001$ ), crossing the predefined margin for significance at the planned interim analysis (data maturity $68 \%$ ). At this interim analysis, OS was immature, but the 1 -year survival rate favored the pyrotinib combination (91.3 vs. $77.4 \%)$.

Treatment-related AEs of grade $\geq 3$ as well as treatment-related serious AEs were observed more frequently in the pyrotinib-combination group (57.5 vs. $34.1 \%$ and 6.0 vs. $1.5 \%$, respectively). Dose modifications ( 47.0 vs. $33.3 \%$ ) and treatment interruptions (61.9 vs. $48.5 \%)$ due to AEs occurred more often with pyrotinib. AEs leading to any form of treatment discontinuation were noted in 4 (3.0\%) patients in the pyrotinib arm and $3(2.3 \%)$ in the lapatinib arm. The most common grade $\geq 3$ treatmentrelated $\mathrm{AE}$ was diarrhea (30.6\% in the pyrotinib-combination group vs. $8.3 \%$ in the lapatinib-combination group), leading to 1 discontinuation in the control arm. Treatment-related AEs led to death in 1 patient in the lapatinib group [42].

\section{Discussion}

Based on the promising results in later lines of therapy, some of the drugs presented in this review are being studied in earlier stages of treatment. However, with the results still pending, pertuzumab and trastuzumab combined with chemotherapy, preferably taxane-based, followed by T-DM1, remains the current standard of care in the first- and second-line treatment of advanced or metastatic HER2-positive breast cancer, even though this sequence has not been formally investigated in a randomized controlled trial. 
Table 1. Overview of clinical trials for the treatment of advanced or metastatic breast cancer

\begin{tabular}{|c|c|c|c|c|c|c|c|c|c|}
\hline Line & Trial & Phase & Treatment & $\begin{array}{l}\text { Prior } \\
\text { pertuzumab, } \\
\%\end{array}$ & $\begin{array}{l}\text { Prior } \\
\text { T-DM1, } \\
\%\end{array}$ & $\begin{array}{l}\text { Hazard } \\
\text { ratio } \\
\text { PFS }\end{array}$ & $\begin{array}{l}\text { Median PFS, } \\
\text { months }\end{array}$ & $\begin{array}{l}\text { Hazard } \\
\text { ratio } \\
\text { OS }\end{array}$ & $\begin{array}{l}\text { Median OS, } \\
\text { months }\end{array}$ \\
\hline \multirow[t]{4}{*}{1} & CLEOPATRA $[7,8]$ & 3 & $\begin{array}{l}\text { docetaxel }+\mathrm{T}+\mathrm{PBO} \\
\text { vs. docetaxel }+\mathrm{T}+\mathrm{P}\end{array}$ & 0 & 0 & 0.62 & 18.5 & 0.66 & 57.1 \\
\hline & PERTAIN [12] & 2 & $\mathrm{AI}+\mathrm{T}+/-\mathrm{P}$ & 0 & 0 & 0.65 & 18.9 & - & - \\
\hline & VELVET [9] & 2 & vinorelbine $+\mathrm{T}+\mathrm{P}$ & 0 & 0 & - & 14.3 & - & - \\
\hline & PERUSE [11] & $3 \mathrm{~b}$ & taxane $+\mathrm{T}+\mathrm{P}$ & 0 & 0 & - & $\begin{array}{l}19.6 \text { (docetaxel) } \\
23.0 \text { (paclitaxel) } \\
18.1 \text { (nab-paclitaxel) }\end{array}$ & - & - \\
\hline \multirow[t]{4}{*}{2} & PHEREXA $[10,43]$ & 3 & $\mathrm{C}+\mathrm{T}+/-\mathrm{P}$ & 0 & 0 & $0.82^{*}$ & 11.1 & - & 37.3 \\
\hline & EMILIA $[18,19]$ & 3 & $\begin{array}{l}\text { T-DM1 vs } \\
\mathrm{C}+\mathrm{L}\end{array}$ & - & 0 & 0.65 & 9.6 & 0.68 & 29.9 \\
\hline & EGF100151 [44, 45] & 3 & $\mathrm{C}+/-\mathrm{L}$ & 0 & 0 & 0.47 & 8.4 & $0.87^{*}$ & $18.75^{* * *}$ \\
\hline & PHOEBE [40]** & 3 & $\begin{array}{l}\text { C + Py vs } \\
C+L\end{array}$ & - & - & 0.39 & 12.5 & - & - \\
\hline \multirow[t]{5}{*}{$>2$} & TH3RESA $[46,47]$ & 3 & T-DM1 vs TPC & - & 0 & 0.53 & 6.2 & 0.68 & 22.7 \\
\hline & HER2CLIMB [22] & 3 & $\begin{array}{l}\mathrm{C}+\mathrm{T}+\mathrm{Tu} v \mathrm{~s} \\
\mathrm{C}+\mathrm{T}+\mathrm{PBO}\end{array}$ & 99.7 & 100 & 0.54 & 7.8 & 0.66 & 21.9 \\
\hline & NALA [26] & 3 & $\mathrm{C}+\mathrm{N}$ vs $\mathrm{C}+\mathrm{L}$ & 42.2 & 54.3 & 0.76 & 5.6 & $0.88^{*}$ & 21.0 \\
\hline & SOPHIA $[29,30]$ & 3 & $\mathrm{M}+\mathrm{TPC}$ vs $\mathrm{T}+\mathrm{TPC}$ & 100 & 91.2 & 0.76 & 5.8 & $0.89^{*}$ & 21.6 \\
\hline & $\begin{array}{l}\text { DESTINY-Breast01 } \\
\text { [37] }\end{array}$ & 2 & T-DXd & 65.8 & 100 & - & 16.4 & - & - \\
\hline
\end{tabular}

* Statistically not significant; ${ }^{* *} 45.5 \%$ of the entire population were treated in a 2 nd-line setting, representing the largest proportion; ${ }^{* * *}$ median OS was 75.0 weeks, following the assumption that 4 weeks represent 1 month. OS, overall survival; PFS, progression-free survival; T, trastuzumab; PBO, placebo; $\mathrm{P}$, pertuzumab; AI, aromatase inhibitor; C, capecitabine; L, lapatinib; Py, pyrotinib; TPC, treatment of physician's choice; Tu, tucatinib; N, neratinib; M, margetuximab.

For the third line of therapy, following the abovementioned standard, tucatinib, in combination with trastuzumab and capecitabine, should be considered the preferred option for 3 major reasons. First of all, all patients had to be pretreated with pertuzumab and T-DM1 prior to enrollment, so this study population is most representative of a patient entering the third line of therapy in our routine practice. Second of all, the addition of tucatinib resulted not only in a statistically significant and clinically meaningful improvement in PFS, HER2CLIMB is the only trial demonstrating a significant prolongation of OS in patients this heavily pretreated. And finally, HER2CLIMB is the only randomized phase 3 trial so far which included patients with untreated and progressive CNS metastases, showing a meaningful improvement in this very challenging subset of patients.

The results of NALA must be interpreted with caution and might not be applicable to our routine practice because, unlike HER2CLIMB, only a minor proportion of the included patients had been pretreated with both pertuzumab and T-DM1. Neratinib compared to lapatinib, both combined with capecitabine, improved PFS and the cumulative incidence of interventions for CNS disease but not OS, at the cost of an almost doubling of grade 3 diarrhea, despite mandatory antidiarrheal prophylaxis in the neratinib arm. Therefore, treatment selection should be individualized by weighing the risk of CNS progression and the risk of intolerable diarrhea.

However, as there are neither data on the sequential use of HER2-targeting TKIs nor on re-exposure to capecitabine in this setting, neratinib and lapatinib probably will not be routinely used immediately following the preferable tucatinib-combination therapy. Studies of either neratinib or lapatinib combined with capecitabine or another cytotoxic single agent in patients pretreated with pertuzumab, T-DM1, and tucatinib, are warranted.

As pyrotinib was only studied in Chinese patients who had not been routinely exposed to either pertuzumab or T-DM1, this TKI will play no role in the treatment of patients in regions where standard first- and second-line treatment is available to every patient.

Most of the patients that were enrolled in SOPHIA, the trial investigating margetuximab in combination with the physician's choice of chemotherapy, had also received pertuzumab and T-DM1 prior to inclusion. However, despite significantly improving PFS, no significant differ- 
ence in OS, the trial's coprimary end point, could be demonstrated at the first and second interim analysis. With the final results pending, a definite positioning of margetuximab in the treatment landscape of metastatic HER2-positive breast cancer cannot be determined at the moment.

Although the efficacy of T-DXd appeared remarkable, it must be kept in mind that DESTINY-Breast01 was a nonrandomized, phase 2 trial. T-DXd should thus be kept for heavily pretreated patients in later lines of therapy at the moment. Management of ILD might be challenging in routine practice unless surveillance and treatment strategies are developed.
The main results of the trials presented in this review and other studies that largely inform today's treatment decisions for the management of HER2-positive metastatic breast cancer are summarized in Table 1.

\section{Conflict of Interest Statement}

The author has no conflicts of interest to declare.

\section{Funding Sources}

There was no funding.

\section{References}

1 Franklin MC, Carey KD, Vajdos FF, Leahy DJ, de Vos AM, Sliwkowski MX. Insights into ErbB signaling from the structure of the ErbB2-pertuzumab complex. Cancer Cell. 2004 Apr;5(4):317-28.

2 Agus DB, Akita RW, Fox WD, Lewis GD, Higgins B, Pisacane PI, et al. Targeting ligandactivated ErbB2 signaling inhibits breast and prostate tumor growth. Cancer Cell. 2002 Aug;2(2):127-37.

3 Baselga J, Swain SM. Novel anticancer targets: revisiting ERBB2 and discovering ERBB3. Nat Rev Cancer. 2009 Jul;9(7):463-75.

4 Lee-Hoeflich ST, Crocker L, Yao E, Pham T, Munroe X, Hoeflich KP, et al. A central role for HER3 in HER2-amplified breast cancer: implications for targeted therapy. Cancer Res. 2008 Jul;68(14):5878-87.

5 Scheuer W, Friess T, Burtscher H, Bossenmaier B, Endl J, Hasmann M. Strongly enhanced antitumor activity of trastuzumab and pertuzumab combination treatment on HER2-positive human xenograft tumor models. Cancer Res. 2009 Dec;69(24):9330-6.

6 Baselga J, Cortés J, Kim SB, Im SA, Hegg R, Im YH, et al.; CLEOPATRA Study Group. Pertuzumab plus trastuzumab plus docetaxel for metastatic breast cancer. N Engl J Med. 2012 Jan;366(2):109-19.

7 Swain SM, Kim SB, Cortés J, Ro J, Semiglazov $\mathrm{V}$, Campone $\mathrm{M}$, et al. Pertuzumab, trastuzumab, and docetaxel for HER2-positive metastatic breast cancer (CLEOPATRA study): overall survival results from a randomised, double-blind, placebo-controlled, phase 3 study. Lancet Oncol. 2013 May;14(6):461-71.

8 Swain SM, Miles D, Kim SB, Im YH, Im SA, Semiglazov V, et al.; CLEOPATRA study group. Pertuzumab, trastuzumab, and docetaxel for HER2-positive metastatic breast cancer (CLEOPATRA): end-of-study results from a double-blind, randomised, placebocontrolled, phase 3 study. Lancet Oncol. 2020 Apr;21(4):519-30.

9 Perez EA, López-Vega JM, Petit T, Zamagni C, Easton V, Kamber J, et al. Safety and efficacy of vinorelbine in combination with pertuzumab and trastuzumab for first-line treatment of patients with HER2-positive locally advanced or metastatic breast cancer: VELVET Cohort 1 final results. Breast Cancer Res. 2016 Dec;18(1):126.

10 Urruticoechea A, Rizwanullah M, Im SA, Ruiz AC, Láng I, Tomasello G, et al. Randomized Phase III Trial of Trastuzumab Plus Capecitabine with or without Pertuzumab in Patients with Human Epidermal Growth Factor Receptor 2-Positive Metastatic Breast Cancer Who Experienced Disease Progression during or after Trastuzumab-Based Therapy. J Clin Oncol. 2017 Sep;35(26):3030-8.

11 Bachelot T, Ciruelos E, Schneeweiss A, Puglisi F, Peretz-Yablonski T, Bondarenko I, et al.; PERUSE investigators. Preliminary safety and efficacy of first-line pertuzumab combined with trastuzumab and taxane therapy for HER2-positive locally recurrent or metastatic breast cancer (PERUSE). Ann Oncol. 2019 May;30(5):766-73.

12 Rimawi M, Ferrero JM, de la Haba-Rodriguez J, Poole C, De Placido S, Osborne CK, et al.; PERTAIN Study Group. First-Line Trastuzumab plus an Aromatase Inhibitor, with or without Pertuzumab, in Human Epidermal Growth Factor Receptor 2-Positive and Hormone Receptor-Positive Metastatic or Locally Advanced Breast Cancer (PERTAIN): A Randomized, Open-Label Phase II Trial. J Clin Oncol. 2018 Oct;36(28):2826-35.

13 Cardoso F, Senkus E, Costa A, Papadopoulos E, Aapro M, André F, et al. 4th ESO-ESMO International Consensus Guidelines for Advanced Breast Cancer (ABC 4). Ann Oncol. 2018 Aug;29(8):1634-57.

14 Giordano SH, Temin S, Chandarlapaty S, Crews JR, Esteva FJ, Kirshner JJ, et al. Systemic Therapy for Patients with Advanced Human Epidermal Growth Factor Receptor 2-Positive Breast Cancer: ASCO Clinical Practice Guideline Update. J Clin Oncol. 2018 Sep;36(26):2736-40.

15 Lewis Phillips GD, Li G, Dugger DL, Crocker LM, Parsons KL, Mai E, et al. Targeting HER2-positive breast cancer with trastuzumab-DM1, an antibody-cytotoxic drug conjugate. Cancer Res. 2008 Nov;68(22):9280-90.

16 Junttila TT, Li G, Parsons K, Phillips GL, Sliwkowski MX. Trastuzumab-DM1 (T-DM1) re- tains all the mechanisms of action of trastuzumab and efficiently inhibits growth of lapatinib insensitive breast cancer. Breast Cancer Res Treat. 2011 Jul;128(2):347-56.

17 Kovtun YV, Audette CA, Ye Y, Xie H, Ruberti MF, Phinney SJ, et al. Antibody-drug conjugates designed to eradicate tumors with homogeneous and heterogeneous expression of the target antigen. Cancer Res. $2006 \mathrm{Mar}$; 66(6):3214-21.

18 Verma S, Miles D, Gianni L, Krop IE, Welslau M, Baselga J, et al.; EMILIA Study Group. Trastuzumab emtansine for HER2-positive advanced breast cancer. N Engl J Med. 2012 Nov;367(19):1783-91.

19 Diéras V, Miles D, Verma S, Pegram M, Welslau M, Baselga J, et al. Trastuzumab emtansine versus capecitabine plus lapatinib in patients with previously treated HER2-positive advanced breast cancer (EMILIA): a descriptive analysis of final overall survival results from a randomised, open-label, phase 3 trial. Lancet Oncol. 2017 Jun;18(6):732-42.

20 Conte B, Fabi A, Poggio F, Blondeaux E, Dellepiane C, D'Alonzo A, et al.; Gruppo Italiano Mammella (GIM) study group. T-DM1 Efficacy in Patients with HER2-positive Metastatic Breast Cancer Progressing after a Taxane Plus Pertuzumab and Trastuzumab: An Italian Multicenter Observational Study. Clin Breast Cancer. 2020 Apr;20(2):e181-7.

21 Prete SD, Montella L, Arpino G, Buono G, Buonerba C, Dolce P, et al. Second line trastuzumab emtansine following horizontal dual blockade in a real-life setting. Oncotarget. 2020 Jun;11(22):2083-91.

22 Moulder SL, Borges VF, Baetz T, Mcspadden T, Fernetich G, Murthy RK, et al. Phase I study of ONT-380, a HER2 Inhibitor, in patients with HER2+-advanced solid tumors, with an expansion cohort in HER2+ metastatic breast cancer (MBC). Clin Cancer Res. 2017 Jul;23(14):3529-36.

23 Pheneger T, Bouhana K, Anderson D, Garrus $\mathrm{J}$, Ahrendt $\mathrm{K}$, Allen $\mathrm{S}$, et al. In vitro and in vivo activity of ARRY-380: a potent, small molecule inhibitor of ErbB2. Cancer Res. 2009; 69(9 Suppl): 1795 . 
24 Murthy RK, Loi S, Okines A, Paplomata E, Hamilton E, Hurvitz SA, et al. Tucatinib, Trastuzumab, and Capecitabine for HER2Positive Metastatic Breast Cancer. N Engl J Med. 2020 Feb;382(7):597-609.

25 Murthy R, Borges VF, Conlin A, Chaves J, Chamberlain M, Gray T, et al. Tucatinib with capecitabine and trastuzumab in advanced HER2-positive metastatic breast cancer with and without brain metastases: a non-randomised, open-label, phase $1 \mathrm{~b}$ study. Lancet Oncol. 2018 Jul;19(7):880-8.

26 Lin NU, Borges V, Anders C, Murthy RK, Paplomata E, Hamilton E, et al. Intracranial Efficacy and Survival with Tucatinib Plus Trastuzumab and Capecitabine for Previously Treated HER2-Positive Breast Cancer with Brain Metastases in the HER2CLIMB Trial. J Clin Oncol. 2020 Aug;38(23):2610-9.

27 Rabindran SK, Discafani CM, Rosfjord EC, Baxter M, Floyd MB, Golas J, et al. Antitumor activity of HKI-272, an orally active, irreversible inhibitor of the HER-2 tyrosine kinase. Cancer Res. 2004 Jun;64(11):3958-65.

28 Saura C, Oliveira M, Feng YH, Dai MS, Chen SW, Hurvitz SA, et al.; NALA Investigators. Neratinib Plus Capecitabine versus Lapatinib plus Capecitabine in HER2-Positive Metastatic Breast Cancer Previously Treated with $\geq 2$ HER2-Directed Regimens: phase III NALA trial. J Clin Oncol. 2020 Sep;38(27): 3138-49.

29 Nordstrom JL, Gorlatov S, Zhang W, Yang Y, Huang L, Burke S, et al. Anti-tumor activity and toxicokinetics analysis of MGAH22, an anti-HER2 monoclonal antibody with enhanced $F c \gamma$ receptor binding properties. Breast Cancer Res. 2011;13(6):R123.

30 Nordstrom JL, Muth J, Erskine CL, Sanders C, Yusko EC, Emerson RO, et al. High frequency of HER2-specific immunity observed in patients (pts) with HER2+ cancers treated with margetuximab $(\mathrm{M})$, an Fc-enhanced antiHER2 monoclonal antibody (mAb). Annual Meeting of the American Society of Clinical Oncology; 2019; Chicago.

31 Rugo HS, Im SA, Wright GS, Escriva-de-Romani S, DeLaurentiis M, Cortes J, et al. SOPHIA primary analysis: a phase 3 (P3) study of margetuximab $(\mathrm{M})+$ chemotherapy $(\mathrm{C})$ versus trastuzumab $(\mathrm{T})+\mathrm{C}$ in patients (pts) with HER2+ metastatic (met) breast cancer (MBC) after prior anti-HER2 therapies (Tx). J Clin Oncol. 2019;37(15_suppl):1000.
32 Rugo HS, Im SA, Cardoso F, Cortes J, Curigliano G, Pegram MD, et al. Phase 3 SOPHIA study of margetuximab + chemotherapy vs trastuzumab + chemotherapy in patients with HER2+ metastatic breast cancer after prior anti-HER2 therapies: second interim overall survival analysis. Cancer Res. 2020;80(4 Suppl):GS1-02.

33 Doi T, Shitara K, Naito Y, Shimomura A, Fujiwara Y, Yonemori K, et al. Safety, pharmacokinetics, and antitumour activity of trastuzumab deruxtecan (DS-8201), a HER2-targeting antibody-drug conjugate, in patients with advanced breast and gastric or gastrooesophageal tumours: a phase 1 dose-escalation study. Lancet Oncol. 2017 Nov; 18(11): 1512-22.

34 Aggarwal N, Sloane BF. Cathepsin B: multiple roles in cancer. Proteomics Clin Appl. 2014 Jun;8(5-6):427-37.

35 Ruan J, Zheng H, Fu W, Zhao P, Su N, Luo R. Increased expression of cathepsin L: a novel independent prognostic marker of worse outcome in hepatocellular carcinoma patients. PLoS One. 2014 Nov;9(11):e112136.

36 Nakada T, Sugihara K, Jikoh T, Abe Y, Agatsuma T. The Latest Research and Development into the Antibody-Drug Conjugate, [fam-] Trastuzumab Deruxtecan (DS-8201a), for HER2 Cancer Therapy. Chem Pharm Bull (Tokyo). 2019;67(3):173-85.

37 Ogitani Y, Aida T, Hagihara K, Yamaguchi J, Ishii C, Harada N, et al. DS-8201a, a novel HER2-targeting ADC with a novel DNA topoisomerase I inhibitor, demonstrates a promising antitumor efficacy with differentiation from T-DM1. Clin Cancer Res. 2016 Oct;22(20):5097-108.

38 Ogitani Y, Hagihara K, Oitate M, Naito H, Agatsuma T. Bystander killing effect of DS8201a, a novel anti-human epidermal growth factor receptor 2 antibody-drug conjugate, in tumors with human epidermal growth factor receptor 2 heterogeneity. Cancer Sci. 2016 Jul; 107(7):1039-46.

39 Modi S, Saura C, Yamashita T, Park YH, Kim SB, Tamura K, et al.; DESTINY-Breast01 Investigators. Trastuzumab Deruxtecan in Previously Treated HER2-Positive Breast Cancer. N Engl J Med. 2020 Feb;382(7):610-21.
40 Jerusalem G, Park YH, Yamashita T, Hurvitz SA, Chen S, Cathcart J, et al. 1380 CNS metastases in HER2-positive metastatic breast cancer treated with trastuzumab deruxtecan: DESTINY-Breast01 subgroup analyses. Ann Oncol. 2020;31(2 Suppl):S63-4.

41 Zhu Y, Li L, Zhang G, Wan H, Yang C, Diao $\mathrm{X}$, et al. Metabolic characterization of pyrotinib in humans by ultra-performance liquid chromatography/quadrupole time-of-flight mass spectrometry. J Chromatogr B Analyt Technol Biomed Life Sci. 2016 Oct;10331034:117-27.

42 Xu B, Yan M, Ma F, Hu XC, Feng JF, Ouyang $\mathrm{Q}$, et al. Pyrotinib or lapatinib plus capecitabine for HER2+ metastatic breast cancer (PHOEBE): A randomized phase III trial. J Clin Oncol. 2020;38:15_suppl:1003.

43 Urruticoechea A, Rizwanullah M, Im SA, Ruiz ACS, Láng I, Tomasello G, et al. Final overall survival (OS) analysis of PHEREXA: A randomized phase III trial of trastuzumab $(\mathrm{H})$ + capecitabine $(\mathrm{X}) \pm$ pertuzumab $(\mathrm{P})$ in patients with HER2-positive metastatic breast cancer (MBC) who experienced disease progression during or after H-based therapy. J Clin Oncol. 2018;36:15_suppl:1013.

44 Geyer CE, Forster J, Lindquist D, Chan S, Romieu CG, Pienkowski T, et al. Lapatinib plus capecitabine for HER2-positive advanced breast cancer. N Engl J Med. 2006;355:273343.

45 Cameron D, Casey M, Oliva C, Newstat B, Imwalle B, Geyer CE. Lapatinib plus capecitabine in women with HER-2-positive advanced breast cancer: final survival analysis of a phase III randomized trial. Oncologist. 2010;15(9):924-34.

46 Krop IE, Kim SB, González-Martín A, LoRusso PM, Ferrero JM, Smitt M, et al.; TH3RESA study collaborators. Trastuzumab emtansine versus treatment of physician's choice for pretreated HER2-positive advanced breast cancer (TH3RESA): a randomised, open-label, phase 3 trial. Lancet Oncol. 2014 Jun;15(7): 689-99.

47 Krop IE, Kim SB, Martin AG, LoRusso PM, Ferrero JM, Badovinac-Crnjevic $\mathrm{T}$, et al. Trastuzumab emtansine versus treatment of physician's choice in patients with previously treated HER2-positive metastatic breast cancer (TH3RESA): final overall survival results from a randomised open-label phase 3 trial. Lancet Oncol. 2017 Jun;18(6):743-54. 